\title{
INTERSECCIONALIDAD Y FRONTERAS SOCIALES: GÉNERO Y CLASE SOCIAL EN EL MERCADO LABORAL CHILENO*
}

\author{
Claudia Mora \\ Centro Sociedad Tecnológica y Futuro Humano, \\ Universidad Mayor, Chile
}

\begin{abstract}
Resumen: Este artículo discute el aporte de la perspectiva interseccional al análisis de la desigualdad social, tomando como caso de estudio la convergencia del género y la clase social en el mercado laboral chileno. Si bien los estudios sobre la desigualdad han desarrollado extensamente el conocimiento de los procesos de reproducción del género y la clase como categorías de análisis independientes, este trabajo destaca la intersección de desigualdades como una contribución a la comprensión de desigualdades complejas. El artículo plantea el aporte metodológico del concepto de fronteras sociales en el estudio de la interseccionalidad y argumenta que la desigualdad interseccional es revelada en las prácticas cotidianas de diferenciación de género y clase social en el mercado del trabajo.
\end{abstract}

Palabras Clave: interseccionalidad, desigualdad social, género, clase social, fronteras sociales, mercado del trabajo

RECIBIDO: julio 2019 / ACEPTADO: septiembre 2019

Claudia Mora es PhD en Sociología. Investigadora del Centro Sociedad Tecnológica y Futuro Humano de la Facultad de Humanidades, Universidad Mayor, Santiago, Chile. Email: claudia.mora@umayor.cl.

* Agradecimientos a Fondecyt 1130779. 


\section{INTERSECTIONALITY AND SOCIAL BOUNDARIES: GENDER AND SOCIAL CLASS IN THE CHILEAN LABOR MARKET}

ABSTRACT: This article discusses the intersectional approach to social inequality, taking the case-study of the convergence of gender and social class in the Chilean labor market. Although the literature on inequality has extensively developed the understanding of gender and class processes of social reproduction as independent categories of analysis, this article highlights the intersection of inequalities as an important contribution to the understanding of complex inequalities. This study contends the methodological contribution of the notion of social boundaries and argues that intersectional inequalities are revealed in the day to day practices of gender and class differentiation in the labor market.

KeYwords: intersectionality, social inequality, gender, social class, social boundaries, labor market

RECEIVED: July 2019 / ACCEPTED: September 2019

\section{INTRODUCCIÓN}

E 1 estudio de la desigualdad social en Chile ha abordado la estratificación principalmente en virtud de la clase social, de acuerdo a su caracterización, formas de subjetivación, al efecto que de ella deriva en las condiciones de vida de las personas, así como también ha abordado la estamentalización de la clase versus perspectivas de movilidad social, entre otros aspectos (Gayo et al. 2013; Castillo 2011; Barozet y Espinoza 2009; Méndez 2008; Torche y Wormald 2007). En la última década han proliferado nuevas aproximaciones a este clásico tema, por ejemplo, en nuevos enfoques en torno a la espacialización de la clase y al 'enclasamiento' del espacio (Matus 2017; Méndez y Barozet 2012; Contreras-Gatica 2011), desde las perspectivas de las bases geográficas de las clasificaciones, las élites y la reproducción social de privilegios, por mencionar algunas (Pelfini 2014; Güell y Joignant 2011).

La literatura acerca de la desigualdad también ha ampliado su espectro de estudio hacia otros patrones de distribución dispar de recursos, incluyendo - en la última década - procesos de exclusión por género en el mercado del trabajo (Undurraga 2018; Cárdenas et al. 2014; Mora 2013; Guzmán 2009), así como la ‘racialización’ de grupos 
migratorios (Tijoux y Sir 2015; Stefoni 2014; Thayer 2013). Un eje común en estos trabajos ha sido el análisis de las categorías de clase social, género y raza como resultado de procesos micro y macrosociales que organizan la distribución desigual de recursos materiales y simbólicos como ingresos, estatus y poder, y la adquisición de capitales necesarios para acceder a ellos. Por lo general, estos análisis han considerado una dimensión específica de análisis, ya sea género, clase social, raza/ etnia, etc.

La desigualdad cruzada por distintas categorías ha sido más bien abordada en tanto realidad donde se dan procesos independientes y paralelos de subordinación. Por ejemplo, en el estudio de mujeres de escasos recursos, a menudo este se plantea como un intento por explicar los efectos materiales de la dimensión de género, más que como una reflexión sobre la clase social y el género en un espacio concurrente de desigualdad; vale decir, en la intersección de estas categorías y en su mutua incumbencia.

La simultaneidad de desigualdades sociales o 'interseccionalidad', término acuñado por la jurista estadounidense Kimberlé Crenshaw en 1989, inicialmente planteó el problema de la convergencia de desigualdades a través de una crítica al uso político del género desprovisto de la dimensión racial, es decir, como crítica a la unidimensionalidad del análisis de la desigualdad social. Crenshaw subrayó el efecto conjunto del género y la raza en cuanto categorías de exclusión, argumentando que la posición particular de la intersección raza/clase no era protegida por las leyes contra la discriminación de Estados Unidos. En tres casos distintos, Crenshaw mostró que el criterio de protección legal se centraba en el género o en la raza, sin embargo, en los lugares de empleo demandados por discriminación se contrataba a mujeres blancas y/o a hombres afroamericanos. De este modo se cumplían los estándares de protección jurídica y la discriminación de mujeres afrodescendientes quedaba, consecuentemente, sin protección por las leyes de antidiscriminación.

La propuesta de Crenshaw (1989), luego adoptada por Hill-Collins (1998), recogía el análisis de distintas categorías de opresión, intentando caracterizar los efectos materiales y simbólicos en una suma de posiciones de marginación, que Hancock (2007) criticó como una verdadera 'olimpiada de la opresión'. Esta perspectiva aditiva, vastamente desa- 
rrollada en los estudios interseccionales, asumía un cruce de categorías conceptualmente independientes y paralelas, que puede ser resumido como un análisis de género más clase (u otras categorías adicionales de subordinación). La constatación de múltiples categorías de opresión no esclarecía, sin embargo, el significado y los efectos materiales y simbólicos de la convergencia de desigualdades, y tampoco la relación entre distintas posiciones de dominación en la configuración de categorías subordinadas. El análisis interseccional desarrollado en los últimos años responde más bien a las preguntas por el cómo interactúan el género y la clase en todas sus dimensiones - no solo en las relativas a la subordinación-, y por cuáles son los efectos en la distribución de recursos sociales que genera el espacio interseccional que emerge en el cruce de categorías de desigualdad.

En este artículo se destacan algunos de los aportes que ofrece esta perspectiva interseccional al caso de estudio de la desigualdad en el mercado laboral; en particular se analizan la intersección del género y la clase social en el mercado laboral chileno. Se plantea la contribución de un análisis del sentido de microprácticas y sus contextos organizacionales en la producción de diferencias clase/género y el aporte metodológico que resulta de incorporar la noción de fronteras simbólicas en la exploración de la interseccionalidad.

En lo que sigue se describen brevemente el género y la clase social como categorías clave en el análisis del mercado laboral; también se revisa el abordaje intercategorial propuesto por McCall (2005) en cuanto aporte significativo al debate metodológico en el campo. Se argumenta que la desigualdad interseccional es revelada en las prácticas cotidianas de reproducción de fronteras simbólicas y en sus múltiples efectos (Lamont y Molnár 2002; Lamont et al. 2014), por lo que la interseccionalidad de desigualdades se trasluce al explorar fronteras de género y clase social que emergen en las prácticas cotidianas de interacción en el trabajo.

\section{GÉNERO Y CLASE SOCIAL EN EL MERCADO LABORAL}

La pregunta por los efectos del género en los esquemas tradicionales de clase social interroga la neutralidad de la categoría femenino/masculino en la distribución de recursos materiales y en la configuración de la estratificación social. La cuestión refiere al alcance explicativo y 
a la centralidad de la estratificación por clase en la desigualdad social y a la posibilidad de que los esquemas de clase se vean alterados por la atención al género. La perspectiva interseccional intenta responder considerando distintos procesos sociales que configuran conjuntamente posiciones y condiciones de vida diferenciadas, y evidenciando la estructura de posiciones de clase que subyace a la segmentación por género y viceversa - esto es, cómo la posición social de género contenida en la estructura de clase afecta la distribución de recursos en sus distintas clasificaciones.

En la literatura internacional, uno de los ámbitos que ha concentrado el análisis interseccional es el mercado del trabajo, considerado como un espacio primario de producción de desigualdad económica y social (Acker 2006). En Chile, el estudio de la desigualdad en el trabajo se ha abordado principalmente desde una categoría de desigualdad, género o clase social, más que de manera integrativa, lo que permitiría mostrar las posiciones diferenciadas entre mujeres y entre hombres -mujeres privilegiadas en virtud de la clase social u hombres subordinados en virtud de la misma-, y las concentraciones por género en las clasificaciones de clase social. Por ejemplo, las mujeres chilenas se concentran en la clase trabajadora; tienen menores chances de movilidad en la estructura de clase (ENCLA 2014), y tienen empleos de menor calidad que los hombres (Aguilar et al. 2016). Por otra parte, las mujeres de clase alta pueden obviar los obstáculos al acceso y la movilidad laboral, por ejemplo, comprando servicio doméstico en el mercado y privilegiando su capital social (Mora y Blanco 2018; Undurraga 2018). Las trabajadoras de escasos recursos, en cambio, dependen de subsidios $\mathrm{y}$ apoyos estatales para ingresar y permanecer en trabajos remunerados.

La interseccionalidad aborda la manera en que el género constituye la clase social y la clase social al género, explorando, entre otros factores, la vinculación entre la organización de la producción y la organización de la reproducción, como uno de los aspectos que condiciona la concentración de mujeres en los estratos más bajos de la clase social (Mora 2013). La relación producción/reproducción contribuye a explicar la segregación laboral de hombres y mujeres en ciertas ramas de actividad y en ocupaciones con valoración diferenciada, generalmente inferior. Demostrar que las posiciones de clase están cruzadas por el género dirige el análisis hacia la fuente de estas diferenciaciones; por 
ejemplo, hacia las políticas en torno a la maternidad y el cuidado de niños, que condicionan el empleo principalmente de mujeres de escasos recursos; a las culturas organizacionales, que limitan la movilidad laboral de las mujeres y las direccionan hacia ciertas actividades; a las variaciones geográficas, industrias y modos de producción locales; al origen nacional y estatus migratorio que configuran condiciones particulares de vida; y a las interacciones cotidianas que configuran las fronteras de clase y género en contextos institucionales específicos.

Entre las múltiples explicaciones de la diferenciación y segregación por género del mercado laboral chileno, son recurrentes las situadas a nivel individual, por ejemplo, en los estereotipos de empleadores, que actuarían como filtro direccionando hacia tareas 'apropiadas' de acuerdo al género; y en las preferencias de los trabajadores, vinculadas a la socialización por género (Undurraga 2018; Abramo 2006; Selamé 2004). Del mismo modo, la justificación de las diferencias en ingresos apuntaría, entre otros aspectos, al tipo de jornada —en promedio, los hombres trabajan jornadas más extensas y las mujeres en jornadas flexibles-; al nivel de capacitación y experiencia en el empleo (vinculado a lo anterior); y a los efectos de la segmentación ocupacional (Perticará y Bueno 2009; Valenzuela y Reinecke 2000; Guzmán et al. 1999). En muchos casos, las investigaciones tienden a resaltar como explicación a tales diferencias la elección o preferencia individual más que la existencia de lógicas organizacionales en la selección y asignación de beneficios a trabajadores, las que a menudo están informadas por criterios de valor que refieren al tiempo en que se distinguía entre trabajo productivo y trabajo reproductivo (Mora 2013). En la versión estructural, el énfasis ha estado en la organización del trabajo y en la significación masculina de la labor productiva que, finalmente, determina el valor atribuido al trabajo (Mora y Blanco 2018; Arriagada 2013; Guzmán 2009). En otras palabras, en el mercado laboral existe una correlación entre la 'deseabilidad' de un trabajo y el grupo (en este caso, el género) más probable de ocuparlo.

Como es evidente, la vinculación entre actividad y estatus ocupacional obedece no solo al género, sino también a la clase social de los trabajadores. La clase social, abordada estructuralmente, es una categoría de análisis referida a la posición de los individuos en la estructura socioeconómica de un país, de la cual derivan posibilidades desiguales 
de acceso a recursos y oportunidades, condicionando así las trayectorias de vida de las personas (Méndez 2008). Tanto en su inspiración neomarxista como en la neoweberiana, la clase social es definida en virtud de la posición de los sujetos en el espacio económico y en sus relaciones constitutivas: las relaciones de producción (en el primer caso) y las relaciones de empleo (en el segundo). Así, en el modelo de clase social neomarxista de E.O. Wright (1994), los grupos aventajados incluyen a propietarios de los bienes de producción y a trabajadores que acumulan un alto nivel de credenciales y de supervisión sobre otros trabajadores. En el modelo neoweberiano de Erickson y Goldthorpe (1992), las ventajas dependen del sector productivo, autoridad, experticia, naturaleza de los contratos, así como de condiciones de empleo de los trabajadores, entre otros factores.

Las clases sociales configuradas de esta manera permitirían dar cuenta de condiciones de vida, experiencias, actitudes, valores y prácticas comunes a cada segmento (Gayo et al. 2013; Barozet y Espinoza 2009; Méndez 2008; Torche y Wormald 2007). Aun en versiones críticas a la relevancia de la clase en la identidad y acción de los sujetos, las condiciones de vida siguen estando definidas por grupos de ocupación (Weeden y Grusky 2005), dado que el sitio de producción genera grupos homogéneos logrados a través de criterios de selección y condiciones de trabajo comunes. En todos estos modelos, las clases sociales están definidas por distintas relaciones de trabajo que condicionan el acceso a beneficios sociales en un sentido amplio. Como categoría de análisis, la clase evidencia efectos en un extenso rango de condiciones de vida: salud física y mental, nutrición y expectativas de vida, $\mathrm{y}$, entre los efectos más relevantes para el mercado laboral, condiciona el nivel educacional y, consecuentemente, el acceso, condiciones de empleo y trayectorias en el trabajo. La estructura de clases presenta, sin embargo, diferencias significativas entre hombres y mujeres. En efecto, como esquema global de distribución de recursos, las clases sociales están segregadas entre aquellas dominadas por hombres y aquellas dominadas por mujeres, entre otras razones porque los atributos de los modelos de clase social — propiedad, autoridad, autonomía, ingresos - están condicionados por el género.

La desigualdad social —en este caso clase social y género- raramente opera unidimensionalmente. Desigualdades entrecruzadas 
generan espacios de intersección que condicionan trayectorias y subjetividades específicas en el mercado del trabajo. De este modo, la perspectiva interseccional sugiere que el análisis de la desigualdad es más complejo que la demostración de una distribución dispar de recursos materiales y simbólicos basada en variables adscriptivas. Si bien aquel detalla un mapa de ruta en el estudio de procesos de diferenciación, no da cuenta de las particularidades que caracterizan la convergencia de distintos patrones de desigualdad, de sus efectos sobre las condiciones y oportunidades de vida de los sujetos, y, por ende, de sus posibilidades de resistencia y transformación social.

El análisis interseccional permite cuestionar la redistribución de recursos derivada de cambios históricos en el mercado del trabajo como, por ejemplo, el crecimiento del sector servicios y sus efectos en el aumento de la participación laboral de las mujeres, además de evidenciar nuevas fragmentaciones de desigualdad en posiciones de clase común. En efecto, como plantea Oesch (2006), el mercado laboral se caracteriza por clivajes horizontales por género, que pueden ser clasificados en virtud de la lógica de trabajo entre experticia técnica, organizacional (administración del poder organizacional) o interpersonal (atención a demandas personales de la gente). Este criterio discrimina horizontalmente entre grupos que parecen homogéneos respecto de su relación de empleo. Esto es, en cada distinción de acuerdo a lógica de trabajo existe una segregación por género, donde las mujeres, como grupo, se concentran en la lógica de servicio interpersonal, con al menos dos indicadores de diferenciación: la remuneración por el trabajo y las perspectivas de ascenso. Estos indicadores son más bajos en trabajadores de servicios rutinarios, que concentran mayoritariamente a mujeres (Oesch 2006).

Una descripción de fragmentaciones clase/género como la referida más arriba, solo puede surgir al considerar la convergencia de estas desigualdades. En Chile, un aporte al análisis interseccional en el mercado del trabajo es el estudio de Aguilar et al. (2016), el cual demuestra que un modelo de clase social interseccional resulta más robusto al captar diferencias por género en la calidad del empleo. Los autores analizaron la clase social y el género, concluyendo que ambos tienen un efecto significativo sobre la calidad objetiva del empleo y las percepciones de los trabajadores sobre esta; y que la relación entre ser mujer y/o perteneciente a una clase subordinada y tener un empleo de menor calidad, 
es significativa. Los autores plantearon que el poder explicativo de la clase social es mayor para los hombres que para las mujeres, y que ello podría estar relacionado con la masculinización de las posiciones de autoridad en el proceso productivo.

Si bien el estudio no aborda - y no podría hacerlo dada la naturaleza de los datos cuantitativos - los procesos que constituyen experiencias distintas y diferenciadas por género, sí abre preguntas relevantes al estudio de la interseccionalidad al establecer que distintas variables de calidad de empleo son sensibles al género y a la clase. Las explicaciones de estas diferencias podrían ser abordadas cualitativamente, por ejemplo, a través de preguntas sobre las expectativas, trayectorias, y movilidad de hombres y mujeres en distintas clases sociales, explorando la vinculación entre una percepción negativa de calidad de empleo y las experiencias de exclusión de las mujeres en el mercado laboral o, a la inversa, entre la no discriminación y una percepción positiva.

La complejidad de la desigualdad social se muestra en la imbricación entre género y clase social que atraviesa la distribución de recursos en Chile, y que en las últimas dos décadas se ha profundizado en virtud de diferenciaciones por origen nacional y estatus migratorio. Ello desafía a los investigadores de la desigualdad con una complejidad de análisis no abordada cabalmente hasta ahora. Los esquemas tradicionales de caracterización de grupos y categorías de desigualdad (clase, género, raza, etc.) no explican las fragmentaciones grupales que emergen de la intersección de distintas categorías en la configuración de oportunidades y trayectorias de vida en contextos institucionales específicos. Las alternativas metodológicas de la aproximación interseccional son aún escasas, y de su desarrollo depende el potencial aporte de esta perspectiva a la comprensión profunda y multidimensional de las formas en que opera la desigualdad social.

\section{ALTERNATIVAS DE ANÁLISIS INTERSECCIONAL}

En su estudio sobre la complejidad de la desigualdad social, McCall (2005) propone tres aproximaciones analíticas a la interseccionalidad, privilegiando la amplitud y rendimiento teórico de una de ellas, que denomina abordaje intercategorial. Este supone la aceptación provisoria de categorías de desigualdad (por ejemplo, la clase social y el género), 
exclusivamente como instrumentos de análisis que permitan explorar los patrones de su producción y reproducción en un contexto institucional dado. A diferencia de métodos anteriores, el abordaje intercategorial no asume la existencia de categorías sociales de fronteras estables y definidas, sino, más bien, una utilización heurística en que dichas categorías son comparadas en todos los puntos de intersección y no solo en los de subordinación.

Ello significa que el estudio interseccional implica un extenso mapa de puntos de comparación: hombre/mujer, distintas clases sociales y grupos raciales, por ejemplo. En otras palabras, McCall propone explorar todo el rango de dimensiones de diferenciación disponibles —no solo los grupos socialmente excluidos- y todo el rango de categorías pertinentes - de clase, género, raza, orientación sexual, ciudadanía, etc.- para entender los efectos de unas sobre otras en los procesos de reproducción de desigualdades y sus posibles resistencias cotidianas (McCall 2005). Esta aproximación al estudio de la interseccionalidad permite responder a los distintos énfasis planteados con anterioridad: la inclusión en el análisis de categorías de dominación y subordinación; la atención al proceso interactivo de efectos de múltiples categorías de desigualdad; y la identificación de categorías que potencialmente predominan en una institución dada, preguntándose por cómo cada eje de análisis está cruzado por otras formas de exclusión (que pueden estar menos articulados al interior de las instituciones).

Una ventaja de esta propuesta es la necesaria problematización de categorías 'neutras' o invisibilizadas — como la masculinidad, el privilegio de clase o la heterosexualidad-, que no son usualmente integradas en las aproximaciones más conocidas, que ponen el énfasis en el cruce de categorías de subordinación. McCall (2005) recoge la crítica anticategorial a la simplificación de lo social, que supone la aplicación mecánica de modelos de desigualdad disponibles. A esta crítica, que sugiere el análisis de la desigualdad a través de la deconstrucción de categorías sociales, McCall propone su utilización provisoria como instrumento de análisis inicial, para mostrar luego el proceso social de su construcción e imbricación mutua.

El análisis interseccional es fortalecido si, además, son tomadas en cuenta las agendas políticas y los contextos socioeconómicos que afectan la reproducción de desigualdades y sus posibilidades de transforma- 
ción (Walby et al. 2012). Ello sumaría dimensiones pocas veces exploradas por los esquemas predominantes de desigualdad social, a saber, la consideración de normas y culturas organizacionales en la producción y las posibilidades individuales de negociar determinados obstáculos, que resultan en el predominio de ciertos patrones de desigualdad por sobre otros. La atención a los contextos social, político y económico permite explorar relaciones sociales desiguales que pueden ser incipientes, incorporando una visión histórica que permite dilucidar la institucionalización temporal de ciertas relaciones de subordinación o dominación, así como de nuevos entornos y posibilidades de negociación.

En el análisis concreto de la interseccionalidad intercategorial, tomando como puntos de partida modelos de clase, categorías binarias de género $u$ otras clasificaciones en virtud de la raza u origen nacional, la consideración de estos factores nos invita a examinar las relaciones de poder y las resistencias posibles en contextos específicos, posibilitando evidenciar la convergencia de desigualdades en un momento histórico particular y sus posibilidades de subversión.

Otro aporte de la perspectiva interseccional ha sido explorar la interacción entre sistemas de desigualdad, que son transformados en su relación con otros sistemas en una constante adaptación mutua en sus distintos puntos de intersección (Choo y Ferree 2010). Para Choo y Ferree (2010), este es un importante aporte a la investigación comprehensiva de la desigualdad: la interseccionalidad como una perspectiva que considera cada sistema social como contexto de otros sistemas sociales, a los cuales se adapta continuamente. Sin tomar un solo punto de intersección como el predominante en una institución dada, la interseccionalidad, argumentan los autores, debe enfocarse en los efectos de múltiples intercambios entre sistemas de desigualdad, donde pequeños cambios pueden tener un importante efecto en las relaciones sociales desiguales.

Esta prolífica agenda de investigación ilumina distintos caminos en el análisis interseccional, sugiriendo que un punto de partida útil para responder sobre la cuestión de la convergencia de desigualdades es el enfoque en los procesos sociales de reproducción de estas mismas, comenzando por el proceso de construcción de las categorías objeto de análisis, y del contexto sociopolítico en que este se desarrolla. No obstante, un aspecto adicional poco tratado por la literatura existente acerca de la interseccionalidad, es su relación con la identidad de los sujetos y 
su despliegue estratégico en contextos organizacionales específicos (por ejemplo, la performatividad de las estrategias de grupos excluidos para lograr niveles de inclusión social).

Choo y Ferree (2010) argumentan que, sin asumir la estabilidad de identidades, la construcción categorial podría ser explorada en las preguntas por el cómo son reclutados los sujetos que las componen, por cuáles son sus resistencias cotidianas y cuáles son los usos estratégicos de una categoría u otra en contextos específicos. Ello porque, como sugiere Nash (2008), la interseccionalidad debe dar cuenta, además, no solo de la distribución de recursos sociales en virtud de una multiplicidad de categorías entrecruzadas, sino también de las experiencias vividas de identidad, lo que implica incorporar la agencia de los sujetos y el análisis de su capacidad y rango de acción, así como los componentes identitarios que pueden ser desplegados en contextos específicos, dependiendo de su posición social (Nash 2008).

\section{LAS FRONTERAS SOCIALES EN EL ESTUDIO DE LA INTERSECCIONALIDAD}

El abordaje intercategorial propuesto por McCall (2005) puede ser considerado hoja de ruta para el análisis de los procesos de reproducción cotidiana de categorías sociales entrecruzadas. La propuesta de McCall permite atender la fluidez de las relaciones sociales desiguales, a menudo institucionalizadas transitoriamente y enmarcadas en un entorno político e institucional que puede transformar esas relaciones de maneras no predecibles. No obstante, como argumentan Schwalbe et al. (2000), la desigualdad institucionalizada depende finalmente de la interacción cara a cara y de prácticas cotidianas que diferencian a los grupos sociales. El género y la clase - entre otras formas de desigualdad - son etiquetas para referirse a "formas rutinizadas de pensamiento, habla y acción, a través de las cuales algunos intentan dominar y explotar a otros" (Schwalbe et al. 2000, 44), posibilitadas o limitadas por entornos institucionales que reproducen ventajas o desventajas para distintos grupos (Holvino 2010).

Un aporte relevante al estudio cualitativo de la interseccionalidad, adoptando la perspectiva intercategorial, es el enfoque de trabajo de fronteras que elaboran Lamont y Fournier (1992), Lamont y Molnár 
(2002) y Lamont et al. (2014), que aborda el trabajo simbólico de demarcación de fronteras grupales. Este enfoque permite dilucidar prácticas de diferenciación por clase y género, sus significaciones y la incidencia de estas distinciones, elaboradas cotidianamente, en la distribución de recursos sociales. La noción de fronteras implica un análisis tanto de las relaciones sociales desiguales que emergen y se reproducen a través de la diferenciación del otro, como del proceso de definición interna de pertenencia a una comunidad y del reconocimiento externo a ella.

Lamont y otros $(1992,2002,2014)$ muestran cómo las diferenciaciones realizadas por los sujetos separan a los grupos a través de la producción de fronteras simbólicas - distinciones conceptuales entre grupos específicos-, cuyo consenso informa la interacción social. Estas distinciones son clave en la emergencia de fronteras sociales o formas objetivadas de diferencia, cuya producción es lograda, precisamente, a través de la movilización de diferencias o similitudes realizada por distintos grupos en la definición que hacen de sí mismos y, a la vez, de otros, mediada por repertorios culturales disponibles. Así, contextos organizacionales específicos aportan símbolos y significados de los principios de diferenciación utilizables y definen, además, la manera en que son percibidos y experimentados (Wimmer 2008).

En el mercado del trabajo, los principios de diferenciación disponibles que tienden a prevalecer encuentran su fundamento en el capital cultural acumulado, su estatus o su legitimidad. Generalmente, estos coexisten con principios morales que categorizan, por ejemplo, en base al esfuerzo y el compromiso, y con criterios de diferenciación socioeconómicos definidos en torno a signos de pertenencia de clase como, por ejemplo, la membresía en organizaciones de élite (Lamont et al. 1992, 2014; Vallas 2001). Aun cuando los criterios esgrimidos por los sujetos pueden variar, ellos son siempre parte de una lucha cotidiana por definir límites categoriales y asegurar la clausura social (Tilly 2000; Vallas 2001; Wimmer 2008).

Desde la perspectiva de fronteras simbólicas y sociales, la interseccionalidad es abordada a través del análisis de las prácticas cotidianas de inclusión/exclusión, explorando límites categoriales a partir de sus significados y efectos. La perspectiva interseccional se beneficia metodológicamente de esta aproximación al estudio de la desigual- 
dad, en primer lugar, ya que ubica las relaciones sociales en contextos determinados, con proyectos políticos particulares y con prácticas institucionalizadas de repartición de recursos. En segundo lugar, releva interacciones cotidianas en la producción de fronteras sociales, las que recurren a principios de justificación diversos de acuerdo al contexto organizacional. En tercer lugar, establece que tanto el género como la clase se generan de manera relacional, lo que sugiere un conjunto de interacciones y prácticas que resultan en relaciones desiguales cuyos efectos son diferencias materiales, de oportunidades y reconocimiento, entre otros. Por último, establece la importancia de reconocer principios de diferenciación que informan y posibilitan performances o que generan resistencias a ciertas categorizaciones.

Algunas de las preguntas iniciales que podrían guiar la investigación interseccional a través del estudio de fronteras sociales serían: ¿cuáles son las distinciones de clase y género creadas en interacciones y prácticas cotidianas en un contexto institucional específico?; ¿son estas diferenciaciones legitimadas en fuentes comunes, por ejemplo, en principios morales?; ¿cuáles son las consecuencias de esas creencias y prácticas?; ¿cómo se vincula la experiencia de frontera simbólica y social con la producción del yo en un contexto específico? El mapa de categorías entrecruzadas resultante podría revelar vinculaciones y contradicciones micro y macrosociales en la producción de diferencias, contribuyendo a responder cómo se generan ciertos patrones de diferenciación, y a ubicar puntos de intervención y de agencia, retratando además modos de resistencia.

En el caso de estudio del mercado laboral chileno, Mora y Blanco (2018) muestran que las fronteras grupales de clase social y género restringen el acceso a redes y alianzas que se establecen informalmente y que de ellas dependen las posibilidades de reconocimiento y movilidad en este mercado. Dicho trabajo sugiere que las fronteras de género emergen de principios de diferenciación legitimados por un marco normativo que asume el trabajo reproductivo como femenino, y que las fronteras de clase remiten a un acuerdo en torno al valor del origen social y sus disposiciones propias. Ambas diferenciaciones condicionan las trayectorias de los trabajadores de rango medio y bajo, mientras que el género predomina en las trayectorias de trabajadores en cargos de supervisión y gerenciales - consecuencia un tanto evidente del sesgo de 
clase para llegar a ocupar cargos en estos rangos. Esto último no significa que la clase social sea irrelevante en rangos altos, sino, más bien, que esta opera como filtro en el acceso a cargos gerenciales, por lo que la exclusión de género es evidenciada con mayor frecuencia y claridad en la clase gerencial debido a la homogeneidad de clase que la caracteriza.

La intersección clase/género que estructura las jerarquías en el trabajo emerge de las prácticas de diferenciación que realizan los sujetos en tanto trabajadores, y que refieren a estrategias de demostración de pertenencia y/o exclusión de grupos en la distribución de recursos valorados - tanto materiales como simbólicos. En las jerarquías más altas de supervisión y calificación, las fronteras de clase social en el mercado laboral chileno son delimitadas moralmente, por ejemplo, en la adscripción a movimientos conservadores dentro de la religión católica - un tipo de frontera que distinguiría la fibra moral de los miembros de la élite-; culturalmente en el prestigio del alma mater y no solo de un grado académico; y socioeconómicamente en la membresía a grupos exclusivos - country clubs, colegios de élite-, en la residencia en sectores privilegiados, además de manejar un código lingüístico particular, y adoptar un disciplinamiento corporal y una vestimenta señalados por la discreción (Aguilar 2011; Moya y Hernández 2014; PNUD 2017; Gayo et al. 2019).

Las prácticas cotidianas de exclusión por género, por otra parte, emergen con nitidez como la barrera predominante en las trayectorias laborales de las mujeres, como una marca de otredad que debe ser negociada y estrategizada regularmente en el contexto organizacional para ser consideradas 'parte del juego' (Mora y Blanco 2018). El género es diferenciado moralmente en todas las jerarquías de clase, entre otros, en el nivel de compromiso e identificación con el trabajo; $y$, entre las jerarquías más altas, en las capacidades de liderazgo, autonomía y disposición al riesgo. Culturalmente es diferenciado en virtud de 'habilidades innatas', así como de la experiencia y 'racionalidad' de los géneros.

Desde el enfoque de fronteras es posible evidenciar, por ejemplo, cómo la intersección de criterios de diferenciación de clase y género basados en el nombre propio y en 'vestimentas sugerentes' sellan la experiencia de control de trabajadoras no calificadas y de bajo estatus. El nombre propio ya ha sido ampliamente identificado en la literatura chilena como signo de pertenencia de clase (Núñez y Pérez 2007; PNUD 2017), pero en la interacción cotidiana converge, por ejemplo, con otros 
criterios como aquellos referidos al disciplinamiento del cuerpo, donde ambos principios definen un espacio cruzado de subordinación. Estos símbolos de pertenencia en interacciones cotidianas, a pesar de su trivialidad, determinan el acceso a recursos valorados en el trabajo, y son negociados y resistidos performativamente por los trabajadores a través de distintas estrategias de posicionamiento en sus contextos organizacionales (Vallas 2001).

Entender el trabajo de fronteras que es realizado en diferenciaciones por la religión, número de hijos o universidad en la que se estudió - en tanto criterios que definen pertenencia de clase a un grupo que controla recursos-, permite cuestionar las ventajas universales del género masculino como categoría unidimensional - sin considerar la clase social - en la distribución de tales recursos. Asimismo, considerar la dedicación al trabajo, iniciativa y racionalidad como principios de diferenciación de género, sugiere ventajas de clase entre mujeres (y en relación a ciertos grupos de hombres).

Un necesario primer paso para explicar la variación por clase social y género es la atención a configuraciones de desigualdad, vale decir, al análisis de qué relaciones sociales desiguales son relevantes, en qué contexto y con cuáles posibilidades de estrategias y negociación. Ello porque, además, la intersección de clase y género perfila trayectorias laborales heterogéneas relacionadas con marcos normativos y organizacionales (Guzmán 2013) que contribuyen a definir las posibilidades de estrategias y respuestas a clasificaciones hegemónicas - por ejemplo, la posibilidad performativa de las disposiciones de clase o de la masculinidad, a modo de respuesta al posicionamiento subordinado de clase/género (Bergeron et al. 2006). Como resultado de esta heterogeneidad se generan identificaciones fragmentadas en categorías presumiblemente monolíticas — como la clase y el género-, a partir de la interacción y los efectos de múltiples patrones de desigualdad en las oportunidades y condiciones de vida de los sujetos. Es decir, las identidades de los sujetos y sus despliegues estratégicos son preguntas a explorar más que elaboraciones predeterminadas.

Así, la intersección de clase y género se manifiesta en un complejo entramado de principios y efectos que solo pueden ser analizados como categorías cruzadas: un espacio de exclusión que se evidencia en el marco de políticas sociales e institucionales, en formas culturalmente 
aceptadas de interacción, y en las justificaciones organizacionales e individuales a la diferenciación categorial. Este punto de partida puede dar lugar a conceptualizaciones novedosas en torno a la desigualdad y distribución de recursos en virtud del género y la clase, al operar a contrapelo de la evidencia en torno a los efectos de ambas categorías en la distribución de recursos en el trabajo. Por último, la perspectiva interseccional a la desigualdad social releva la convergencia de fronteras simbólicas de clase y género que, como planteó Crenshaw (1989) en su análisis inicial, no puede ser comprendida en un solo eje de diferenciación y exclusión. Ello permite el análisis de la distribución de recursos derivada de constelaciones no siempre claramente visibles o predeterminadas. Los efectos del cruce de desigualdades en las trayectorias de vida, oportunidades y subjetividades conforman, como en estos casos, un campo abierto de exploración en las ciencias sociales.

\section{CONCLUSIONES}

Distintas formas de desigualdad social, como el género y la clase social, han sido extensamente estudiadas en contextos institucionales donde estas desigualdades predominan: la posición de clase en las relaciones de producción y mercado, y el género en las relaciones en torno al cuidado. En entornos institucionales que les son 'ajenos', tanto el estudio de la clase como el del género han transitado a partir de evidenciar sus manifestaciones como desigualdades secundarias hacia un análisis de la desigualdad desde la perspectiva de una de estas categorías, unidimensionalmente. La aproximación interseccional recoge la complejidad de la desigualdad al proponer que distintos sistemas entrecruzados influyen en las posiciones de los sujetos y condicionan sus oportunidades de vida no solo en aspectos materiales, sino que en estatus, reconocimiento y subjetividades de maneras no evidenciadas por los modelos tradicionales de análisis.

La perspectiva interseccional ha generado un debate y área de estudios de largo aliento, desde una primera aproximación 'aditiva' hasta modelos integrados que buscan caracterizar el espacio interseccional y sus efectos en el más amplio rango de esferas -institucional, político, relacional, subjetivo, por mencionar algunos. En este trabajo se han presentado algunos de los aportes relevantes a este debate, entre otros: la 
necesidad de visibilizar categorías neutras en las relaciones de desigualdad; de atender a proyectos políticos diversos que informan lógicas institucionales y que proporcionan un cierto repertorio de acción; y de enfatizar las particularidades de la integración de distintos sistemas de desigualdad. La concentración en procesos de producción de desigualdades interseccionales que se reproducen en el cotidiano, evidencian el establecimiento de lógicas de acceso y distribución de recursos sociales, así como la configuración de identidades y posibilidades de agencia.

Dado que uno de los debates actuales en los estudios de la interseccionalidad es el cómo explorarla, se han desarrollado algunas alternativas metodológicas a su estudio, incorporando la perspectiva interaccional de fronteras sociales en la reproducción de la desigualdad social. Con ello se pretende destacar un mapa de relaciones sociales desiguales que emergen de interacciones cotidianas en un amplio espectro de esferas, los entornos que las facilitan y sus potenciales alcances distributivos e identitarios. La comprobación de que la intersección de desigualdades es discernible principalmente a través del énfasis en la producción cotidiana de fronteras sociales, nos lleva a plantear la necesidad de atender a procesos y relaciones que parecen 'normales' pero que reproducen - no siempre de manera consciente - privilegios y desigualdad.

\section{BIBLIOGRAFÍA}

Abramo, L.W. 2006. Trabajo decente y equidad de género en América Latina. Santiago: Oficina Internacional del Trabajo.

Acker, J. 2006. Inequality Regimes: Gender, Class, and Race in Organizations. Gender \& Society 20(4), 441-464.

Aguilar, O. 2011. Dinero, educación y moral: el cierre social de la elite tradicional chilena (203-240). En Güell, P. y Joignant, A. (eds.), Notables, tecnócratas y mandarines. Elementos de sociología de las elites en Chile (1990-2010). Santiago: Ediciones Universidad Diego Portales.

Aguilar, O., Pérez, P., Ananías, R., Mora, C. y Blanco, O. 2016. Intersección entre la clase y el género y su efecto sobre la calidad del empleo en Chile. Revista de la CEPAL 120, 141-161.

Arriagada, I. 2013. Desigualdades en la familia: trabajo y cuidados en Chile (91112). En Mora, C. (ed.), Desigualdad en Chile: la continua relevancia del género. Santiago: Ediciones Universidad Alberto Hurtado.

Barozet, E. y Espinoza, V. 2009. ¿De qué hablamos cuando decimos 'clase media'? Perspectivas sobre el caso chileno (1-36). El arte de clasificar a los chilenos. 
Enfoques sobre los modelos de estratificación en Chile. Santiago: FocoExpansiva-UDP.

Bergeron, D.M., Block, C.J. y Echtenkamp, A. 2006. Disabling the Able: Stereotype Threat and Women's Work Performance. Human Performance 19(2), 133-158.

Cárdenas, A., Correa, N. y Prado, X. 2014. Segregación laboral y género: tendencias y desafíos relativos al mercado laboral de la salud y la educación en Chile. Polis. Revista Latinoamericana 13(38), 397-441.

Castillo, J.C. 2011. Legitimacy of Inequality in a Highly Unequal Context: Evidence from the Chilean Case. Social Justice Research 24(4), 314-340.

Choo, H.Y. y Ferree, M.M. 2010. Practicing Intersectionality in Sociological Research: A Critical Analysis of Inclusions, Interactions, and Institutions in the Study of Inequalities. Sociological Theory 28(2), 129-149.

Contreras-Gatica, Y. 2011. Elecciones y anclajes residenciales de los nuevos habitantes del centro de Santiago: la experiencia del barrio de Bellas Artes. Revista Transporte y Territorio 4, 59-83.

Crenshaw, K. 1989. Demarginalizing the Intersection of Race and Sex: A Black Feminist Critique of Antidiscrimination Doctrine, Feminist Theory and Antiracist Politics. University of Chicago Legal Forum 140, 139-167.

ENCLA, Encuesta Laboral 2014. Informe de resultados Octava Encuesta Laboral. Santiago: Dirección del Trabajo.

Erikson, R. y Goldthorpe, J.H. 1992. The Constant Flux: A Study of Class Mobility in Industrial Societies. Oxford: Oxford University Press.

Gayo, M., Teitelboim, B. y Méndez, M.L. 2013. Exclusividad y fragmentación: los perfiles culturales de la clase media en Chile. Universum 28(1), 97-128.

Gayo, M., Otero, G. y Méndez, M.L. 2019. Elección escolar y selección de familias: reproducción de la clase media alta en Santiago de Chile. Revista Internacional de Sociología 77(1), 120.

Güell, P. y Joignant, A. (eds.) 2011. Notables, tecnócratas y mandarines: elementos de sociología de las elites en Chile, 1990-2010. Santiago: Universidad Diego Portales.

Guzmán, V., Mauro, A. y Araujo, K. 1999. Trayectorias laborales de mujeres: cambios generacionales en el mercado de trabajo. Santiago: Centro de Estudios de la Mujer.

Guzmán, V. 2009. Instituciones, actores y calidad del empleo. ¿Malos tiempos para un 'buen' trabajo? Calidad del trabajo y género. Cuadernos de Investigación $N^{\circ}$ 4. Santiago: Centro de Estudios de la Mujer.

Guzmán, V. 2013. Discurso de género e institucionalidad pública en Chile 19802010 (199-220). En Mora, C. (ed.), Desigualdad en Chile: la continua relevancia del género. Santiago: Ediciones Universidad Alberto Hurtado.

Hancock, A.M. 2007. When Multiplication doesn't Equal Quick Addition: Examining Intersectionality as a Research Paradigm. Perspectives on Politics 5(1), 63-79. 
Hill-Collins, P. 1998. It's All in the Family: Intersections of Gender, Race, and Nation. Hypatia 13(3), 62-82.

Holvino, E. 2010. Intersections: The Simultaneity of Race, Gender and Class in Organization Studies. Gender, Work \& Organization 17(3), 248-277.

Lamont, M. y Fournier, M. (eds.) 1992. Cultivating Differences: Symbolic Boundaries and the Making of Inequality. Chicago: University of Chicago Press.

Lamont, M. y Molnár, V. 2002. The Study of Boundaries in the Social Sciences. Annual Review of Sociology 28(1), 167-195.

Lamont, M., Beljean, S. y Clair, M. 2014. What is Missing? Cultural Processes and Causal Pathways to Inequality. Socio-Economic Review 12(3), 573-608.

Matus, C. 2017. Estilos de vida e imaginarios urbanos en nuevos residentes de Lastarria y Bellas Artes: el barrio patrimonial como escenario de diversidad, distinción y movilidad. EURE 43(129), 165-186.

McCall, L. 2005. The Complexity of Intersectionality. Signs. Journal of Women in Culture and Society 30(3), 1771-1800.

Méndez, M.L. 2008. Middle Class Identities in a Neoliberal Age: Tensions between Contested Authenticities. The Sociological Review 56(2), 220-237.

Méndez, M.L. y Barozet, E. 2012. Lo auténtico también es público. Comprensión de lo público desde las clases medias en Chile. Polis. Revista Latinoamericana 11(31), 183-202.

Mora, C. 2013. Desigualdad en Chile: la continua relevancia del género. Santiago: Ediciones Universidad Alberto Hurtado.

Mora, C. y Blanco, O. 2018. The Gender Deficit: Everyday Practices of Differentiation in the Chilean Labour Market. Bulletin of Latin American Research 37(3), 291-305.

Moya, E. y Hernández, J. 2014. El rol de los colegios de elite en la reproducción intergeneracional de la elite chilena. Revista Austral de Ciencias Sociales 26, $59-82$.

Nash, J.C. 2008. Re-thinking Intersectionality. Feminist Review 89(1), 1-15.

Núñez, J. y Pérez, G. 2007. Dime cómo te llamas y te diré quién eres: la ascendencia como mecanismo de diferenciación social en Chile. Santiago: Departamento de Economía de la Universidad de Chile.

Oesch, D. 2006. Coming to Grips with a Changing Class Structure: An Analysis of Employment Stratification in Britain, Germany, Sweden and Switzerland. International Sociology 21(2), 263-288.

Pelfini, A. 2014. The Role of Elites in Emerging Societies, or How Established Elites Deal with an Emerging Society: the Case of Business Elites in Contemporary Chile. Transcience 5(1), 53-62.

Perticará, M. y Bueno, I. 2009. Brechas salariales por género en Chile: un nuevo enfoque. Revista de la CEPAL 99, 133-149.

PNUD 2017. Desiguales. Orígenes, cambios y desafios de la brecha social en Chile. Santiago: PNUD. 
Selamé, T. 2004. Mujeres, brechas de equidad y mercado de trabajo. Santiago: Organización Internacional de Trabajo.

Schwalbe, M., Holden, D., Schrock, D., Godwin, S., Thompson, S. y Wolkomir, M. 2000. Generic Processes in the Reproduction of Inequality: An Interactionist Analysis. Social Forces 79(2), 419-452.

Stefoni, C. 2014. La construcción racializada del migrante en el norte de Chile. Revista Crítica y Emancipación 6(11), 581-595.

Thayer, L.E. 2013. Expectativas de reconocimiento y estrategias de incorporación. La construcción de trayectorias degradadas en migrantes latinoamericanos residentes en la Región Metropolitana de Santiago. Polis. Revista Latinoamericana $12(35), 259-285$.

Tijoux, M.E. y Sir, H. 2015. Trayectorias laborales de inmigrantes peruanos en Santiago: el origen de excepción y la persistencia del 'lugar aparte'. Latin American Research Review 50(2), 135-153.

Tilly, C. 2000. La desigualdad persistente. Buenos Aires: Manantial.

Torche, F. y Wormald, G. 2007. Chile, entre la adscripción y el logro (339-387). En Estratificación y movilidad social en América Latina: transformaciones estructurales de un cuarto de siglo. Santiago: CEPAL, LOM Ediciones.

Undurraga, R. 2018. Me preguntaron: ¿quieres tener hijos pronto? (123-161). En Cárdenas, A. y Yévenes, A. (comps.), Mujer(es), familia(s) y trabajo(s). Buenos Aires: Teseo.

Valenzuela, M.E. y Reinecke, G. 2000. ¿Más y mejores empleos para las mujeres?: la experiencia de los países del Mercosur y Chile. Santiago: Organización Internacional del Trabajo.

Vallas, S.P. 2001. Symbolic Boundaries and the New Division of Labor: Engineers, Workers and the Restructuring of Factory Life. Research in Social Stratification and Mobility 18, 3-37.

Walby, S., Armstrong, J. y Strid, S. 2012. Intersectionality: Multiple Inequalities in Social Theory. Sociology 46(2), 224-240.

Weeden, K.A. y Grusky, D.B. 2005. The Case for a New Class Map 1. American Journal of Sociology 111(1), 141-212.

Wimmer, A. 2008. The Making and Unmaking of Ethnic Boundaries: A Multilevel Process Theory. American Journal of Sociology 113(4), 970-1022.

Wright, E.O. 1994. Clases. Madrid: Siglo Veintiuno Editores. EP 